\title{
A Working Population Model for Haemolytic Disease of the Newborn
}

\author{
E. G. KNOX and W. WALKER \\ From the Department of Social Medicine, University of Birmingham, and the \\ Department of Child Health, University of Newcastle upon Tyne
}

This study is based upon three considerations. (1) The sequence of events in the genesis of rhesus haemolytic disease of the newborn is now specifiable with considerable accuracy.

(2) This sequence is too complex for the consequences to be expressed as a simple mathematical function of the premises and parameters upon which they depend.

(3) There is a need for these consequences to be determined accurately and readily. This is partly in order to confirm the premises by comparing their consequences with observations in the population, but more particularly to examine the results of applying preventive techniques of different efficiencies to different parts of the process.

\section{Premises and Parameters}

It has been known for many years that quite a small proportion of infants, who in genetic terms are at risk, in fact develop haemolytic disease of the newborn (HDN). Many possible explanations for this have been entertained at different times, and it would not have been possible 10 years ago to select with any certainty a set of premises on which an adequate model could be based, but more recently it has become possible to specify the timing and nature of all the main determinants of the disease.

Levine discovered in 1943 that the ABO mating types in affected families were not as in the general population, and that there was a deficiency of matings in which the mother had antibodies to the father's group. This has been confirmed many times. It was assumed that the low incidence of $\mathrm{HDN}$ in matings showing paternal/ maternal incompatibility was mediated through a protective fetal/maternal incompatibility, but it was not known whether the protection was effective during the first pregnancy at which an affected

Received March 12, 1968. child might have been expected, or at the previous rhesus positive pregnancy, or other previous pregnancies, or at more than one of these locations. Nevanlinna and Vainio (1956) showed that the proportion of fetal/maternal heterospecificities was lower for the last healthy child than for the first sick one in a family, thus drawing special attention to events before the conception of the first child at risk, but their data did not localize the effect uniquely to the sensitizing pregnancy. A more precise confirmation of their thesis was achieved by Knox and Walker (1957) and Knox, Murray, and Walker (1960) using a double approach. Combined studies of birth rank distributions of first affected infants, and of $\mathrm{ABO}$-heterospecificity frequencies in precisely identified sensitizing pregnancies showed, (a) that powerful environmental determinants operated at a single pregnancy, and (b) that this was the sensitizing pregnancy, and therefore (c) that the outcome of subsequent rhesus positive pregnancies was determined before conception occurred.

Soon after this, Knox, Murray, and Walker (1961), and later Finn et al. (1961), pointed out the possibility of preventing the disease through the immunological destruction of transfused fetal cells. These proposals were developed independently, both teams having presented their unpublished conclusions during the previous year (see Lancet (1960), 1, 526; and Brit. J. prev. soc. Med. (1960) 14, 148, for appropriate notices). Both proposals depended upon the assumption that subsequent events were determined entirely at the sensitizing pregnancy, but each relied upon additional evidence from two different, and in fact complementary, sources. Finn et al. based their suggestions upon their demonstration that fetal blood cells were in fact detectable following delivery in a proportion of women with $\mathrm{ABO}$ homospecific infants, but not when the infant was heterospecific. Knox et al. based their suggestion 
on a direct measurement of the subsequent risk of haemolytic disease when the sensitizing pregnancy and delivery included events which seemed likely to disturb the placental site and thus facilitate fetal/maternal transfusion. In particular, toxaemia of pregnancy, caesarean section, and manual removal of the placenta greatly increased this risk.

Since then, the proposals concerning the mechanism of rhesus iso-immunization have in general been confirmed. Pilkington et al. (1966) demonstrated that the events of pregnancy and delivery which were thought to promote fetal/maternal transfusion did in fact do so. More directly, Clarke et al. (1966) have shown that the development of antibodies six months after delivery, in women suffering fetal/maternal transfusion, can be suppressed through the administration of anti- $\mathrm{D}$ antibodies and the clearance of fetal cells from their circulations.

Nevertheless, a number of problems remain and the ones that particularly concern us here are the best means of identifying those women at risk, and the likely effectiveness of preventive techniques based upon such discrimination and applied in a population context. Immunological suppression appears to be highly effective, approaching $100 \%$ (Clarke, 1968), but available techniques for isolating the women who need to be treated are clearly inefficient. In addition, there are problems concerning the costs and ethics of supplying anti-D antibodies in large quantities, particularly because it may prove necessary to immunize volunteers artificially, or to boost the titres of those already immunized. It has been suggested that haematological investigation and immunological suppression might be limited to first pregnancies in a bid to achieve two normal infants for every $R h$-negative woman. The consequences of this policy, and of other variations, need to be explored. Finally, supposing that over a period of time the incidence of HDN is seen to change, with what confidence can this be attributed to a change in the risk of sensitization, either through the application of preventive methods or from other causes?

\section{The Model}

The relation between the incidence of rhesus HDN in the population, and the risk of rhesus sensitization in an Rh-negative mother with an Rh-positive fetus, depends upon the birth rank distribution of all births in the population, and the frequencies of the different rhesus and $\mathrm{ABO}$ genes.

The relation with the rhesus gene frequencies is complex; the number of rhesus-positive fetuses in rhesus-negative women is determined thereby, but in addition the $R_{2} r$ genotype provides a more powerful antigen than the $R_{1} r$ genotype (Walker, Murray, and Russell, 1957; Murray, Knox, and Walker, 1965). The ABO gene frequencies determine the proportion of heterospecific pregnancies, but again there is a complicating factor in that protection is not complete.

In order to meet these requirements the model was built about the following input parameters.

(a) A statement of the birth rank distribution in the population between ranks 1 and 12 supplied as either absolute numbers, percentages, or proportions.

(b) The gene frequencies $a, b$, and o, supplied as either proportions or percentages.

(c) The main rhesus gene frequencies $R_{1}(C D e)$, $R_{2}(c D E)$, and $r(c d e)$, as either proportions or percentages. The $R_{1}$ gene frequency here is notional and intended to cover all the $R$ genes other than $R_{2}(c D E)$, since the $R_{2} r$ genotype seems to carry a differential sensitization risk.

(d) The risk attributed to an $\mathbf{R}_{2} \mathbf{r}$ pregnancy relative to $1 \cdot 0$ for $R_{1} r$.

(e) The risk attributed to an ABO-heterospecific pregnancy relative to $1 \cdot 0$ for homospecific.

The computation of the accumulating incidence of disease at successive birth ranks does not depend upon simple multiplicative probabilities over the whole population, but upon a separate computation for each of 15 mating types. There are 5 main rhesus mating types ( $\mathrm{rr} \times{ }^{\top} \mathbf{R}_{1} R_{1}, \mathbf{R}_{1} R_{2}, \mathbf{R}_{2} R_{2}, R_{1} r, R_{2} r$ ), and for each of these 3 main ABO mating types according to whether all infants are ABO-heterospecific, or half of them, or none, and each has its own sequence of accumulation of risk with birth rank.

Knox and Walker (1957) showed that the risk of sensitization among susceptible women was substantially the same at each birth rank, but this is not assumed in the model. On the contrary, it is necessary to be able to vary this postulate, partly to permit alternative fittings to data, but mainly to permit modification to represent the effects of prophylactic techniques directed towards particular birth ranks. Therefore the model requires an additional set of input parameters, namely the risks attributable to pregnancies of successive ranks, relative to 1.0 for an unmodified first pregnancy.

The model is written in Fortran IV and is available as an appendix on request from the authors. It can operate in either of two directions. The risk of sensi-

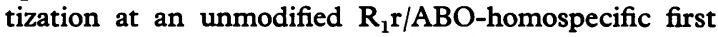
pregnancy may be presented, in which case the consequent birth rank distribution of all affected infants, and of first affected infants, and the total incidence of HDN are computed. Alternatively, the incidence may be supplied, in which case the model operates iteratively until a sensitization risk is derived, which results in the given incidence. The final presentation is based upon this derived result.

The internal operation of the model is based upon computations of mating type frequencies from gene frequencies all of which are standard except those for ABO-heterospecificity, which do not seem previously to have been derived. They are as follows: 
ABO Mating Types

All heterospecific Half heterospecific None heterospecific

The cumulative proportion of sensitized women is derived separately for each of the 15 mating types, and a running total of those who have not:yet borne an affected infant is also maintained. On this basis, together with the rhesus genotype of the father, the numbers of affected infants, and of first affected infants, at each rank, are also accumulated. Specific details are presented in the programme.

\section{Results}

Relation between basic sensitization risk and prevalence. A series of examinations was carried out of the relation between the basic sensitization risk and the incidence which resulted. The model basis was a birth rank distribution as for legitimate deliveries in England and Wales for 1960, with $\mathrm{ABO}$ and Rhesus gene frequencies as for England and Wales. Equal risk was attached to each birth rank, $R_{2} r$ was given a weighting of $1 \cdot 2$, and heterospecific pregnancies a weighting of $0 \cdot 1$. The incidences resulting from allocated basic risks between 0.0 and 0.18 are shown in the Fig. (curve A) where it can be seen that, despite the complexity of the function, the relation is almost linear. An incidence of 6.5 per 1000 infants born corresponds with a sensitization risk of 0.081 in $R_{1}$ r ABO homospecific pregnancies-that is about 1 in 12. In this region of the curve a change of 0.01 in the sensitization risk results in a change of about 0.75 per 1000 in the incidence.

An implication for prophylactic programmes also

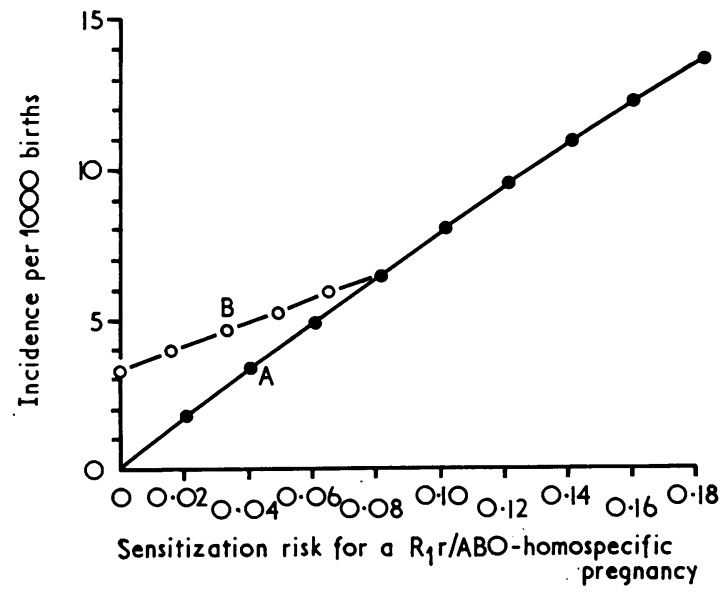

FIG.-Relation between sensitization risk and incidence per 1000 total births, distributed as in England and Wales, 1960. emerges, in that the absolute numerical relation between sensitizations prevented and cases of disease prevented can be calculated. On the basis of the population birth rank distribution for 1960, which is fairly typical of the period, the ratio is almost exactly $1: 1$, provided that the sensitizations saved are proportionately distributed by birth rank.

Birth rank distribution of affected infants. The relation between sensitization risk and subsequent incidence depends upon the premises of the model and so cannot be used to check upon the validity of the premises. However, the birth rank distribution deriving from this estimate of risk does provide an independent means of checking results against observations.

Table I shows data collected for Northumberland and Durham from 1952 to 1966. These results are compared with percentages obtained on the model on the basis of a population birth rank distribution corresponding with that experienced during that time. A local distribution was not available, but data for legitimate births in England and Wales are given by the Registrar General and the exact basis used was the sum of the even years 1952 to 1964 , the latter year having been entered twice pending availability of the 1966 figures.

There are of course problems in comparing expectations with model results because (a) some women are immunized other than by pregnancy, (b) some probably initiate and complete the process during a single pregnancy, (c) the data are unlikely to be $100 \%$ complete, (d) it is possible that the occurrence of an affected child might affect the subsequent pattern of child bearing within a family, and (e) there are problems of uncertainty concerning paternity in a proportion of families.

Some of the problems of comparing expectations with the full birth rank distribution are avoided by comparing only the predictions and observations relating to first affected infants. This was done by Knox and Walker in 1957 using comparatively simple algebraic methods and the comparison is presented again in Table II using the same data, but the new model. In fact the model produced results only slightly different from those of the methods then used.

In both sets of data the correspondences between predicted and observed distributions permit the conclusion that the premises of the model represent the events very well.

Changes in population birth rank distribution. The extent to which changes in the 
TABLE I

Percentage Birth Rank Distribution of All Affected Infants, Northumberland and Durham 1952-1966, on Basis of Total Birth Rank Distribution for those Years in England and Wales

\begin{tabular}{|c|c|c|c|c|c|c|c|c|c|c|c|c|}
\hline & \multicolumn{11}{|c|}{ Rank } & \multirow[b]{2}{*}{ Total } \\
\hline & 1 & 2 & 3 & 4 & 5 & 6 & 7 & 8 & 9 & 10 & over 10 & \\
\hline $\begin{array}{l}\text { Observed distribution } \\
\text { Percentage observed } \\
\text { Percentage predicted }\end{array}$ & $\begin{array}{l}-\star \\
-\star \\
0\end{array}$ & $\begin{array}{c}970 \\
24 \cdot 76 \\
25 \cdot 78\end{array}$ & $\begin{array}{c}1026 \\
26 \cdot 19 \\
26 \cdot 17\end{array}$ & $\begin{array}{c}734 \\
18 \cdot 73 \\
18 \cdot 20\end{array}$ & $\begin{array}{c}466 \\
11 \cdot 89 \\
11 \cdot 43\end{array}$ & $\begin{array}{l}309 \\
7 \cdot 89 \\
7 \cdot 10\end{array}$ & $\begin{array}{l}166 \\
4 \cdot 24 \\
4 \cdot 45\end{array}$ & $\begin{array}{l}101 \\
2 \cdot 58 \\
2 \cdot 74\end{array}$ & $\begin{array}{c}64 \\
1 \cdot 63 \\
1 \cdot 71\end{array}$ & $\begin{array}{l}37 \\
0 \cdot 94 \\
1 \cdot 07\end{array}$ & $\begin{array}{c}45 \\
1 \cdot 15 \\
1 \cdot 36\end{array}$ & $\begin{array}{c}3918 \\
100 \cdot 0\end{array}$ \\
\hline
\end{tabular}

^Rank 1 cases, and rank 2 cases said not to be first in the family, have been excluded for this purpose.

TABLE II

Percentage Birth Rank Distribution of First Affected Infants, Newcastle Region 1947-1956, on Basis of 1951 Population Birth Rank Distribution

\begin{tabular}{l|c|c|c|c|c|c}
\hline & & \multicolumn{3}{|c|}{ Rank } \\
\cline { 2 - 6 } & 1 & 2 & 3 & 4 & 5 & $6+$ \\
\hline $\begin{array}{l}\text { Observed (904 cases) } \\
\text { Model .. }\end{array}$ & $1-\star$ & $43 \cdot 5$ & $28 \cdot 9$ & $13 \cdot 4$ & $8 \cdot 2$ & $6 \cdot 2$ \\
& 0 & $45 \cdot 0$ & $26 \cdot 6$ & $13 \cdot 1$ & $6 \cdot 9$ & $8 \cdot 4$ \\
\hline
\end{tabular}

^Rank 1 cases excluded for this purpose.

birth rank distribution can affect the prevalence of the disease was explored by fixing the basic risk at 0.081 , that is the level which gave an incidence of 6.5 per 1000 in 1960 and by running the model with different birth rank distributions, corresponding with those for England and Wales from 1950, by two-year intervals, to 1964 . The results are shown in Table III. The lowest model incidence during this period was in 1956 , at $6 \cdot 22$ per 1000 , when $40 \%$ of all births were first births, and the highest was 6.73 in 1964 when the proportion of first births was only 36 per cent.

The year 1947 had the highest proportion of first births in recent times, $44.5 \%$, and the resulting incidence of disease on the basis of the model, was $5 \cdot 67$ per 1000 .

Prophylactic procedures. It is evident from the linear relation expressed in the Fig. that any procedure that reduces the risk of sensitization will reduce the prevalence of the disease almost exactly in proportion.

For example Clarke et al. (1966) have assembled results that suggest that if women are selected on the basis of having $\mathbf{5}$ or more fetal cells visible in $\mathbf{5 0}$ low-power fields of a maternal capillary blood smear then approximately $24 \%$ will develop antibodies if they are not treated (19 out of 78 in the experiment), but that almost all of them can be prevented by the administration of anti-D $\gamma$-globulin. The selection level represented by a cell count of 5 or more was not specifically stated, but from a previous study by the same workers (Woodrow et al., 1965) it appears to represent about $10 \%$ of homospecific pregnancies (20 out of 203 in the work referred to). The product of these two percentages specifies the proportion of homospecific pregnancies in which sensitization is prevented by the combination of selection and treatment, that is $2 \%$. We have already seen that about $8 \%$ of such pregnancies will in fact cause sensitization in the absence of treatment, so that the method can expect to effect a reduction of about $2 \cdot 4 / 8 \cdot 1$, that is $36 \%$ of the untreated level in the population as a whole. The model has shown that if this effect were achieved uniformly at all pregnancy ranks, then the incidence too would be reduced by this amount.

The proportion of women selected could be increased by using a less stringent criterion, but the proportion saved from sensitization by treatment might then be expected to fall. For example, Clarke (1968) has recently assembled the results of a set of experiments, some of which use less stringent

TABLE III

Expected Incidence of Haemolytic Disease in Different Years, England and Wales, on Basis of Basic Sensitization Risk of 0.081

\begin{tabular}{ll|l|l|l|l|l|l|l|l|l|}
\hline & & 1950 & 1952 & 1954 & 1956 & 1958 & 1960 & 1962 & 1964 \\
\hline Incidence per 1000 total births & $\ldots$ &. & $6 \cdot 34$ & $6 \cdot 31$ & $6 \cdot 30$ & $6 \cdot 22$ & $6 \cdot 27$ & $6 \cdot 50$ & $6 \cdot 62$ & $6 \cdot 73$ \\
\hline
\end{tabular}


selection criteria than he has used himself, and only $13 \%$ of the controls (76 out of 575 ) developed antibodies. The selection level represented in these studies cannot be specifically stated, but if $20 \%$ of women are selected for treatment (as opposed to $10 \%$ in Clarke et al.'s 1966 study) then the result is a reduction of $31 \%$ in the risk of sensitization over the whole population.

With further development the efficiency of selection by the Kleihauer technique might be improved. However, it is unlikely that the population is so homogeneous in terms of response to stimulus that any particular level of transfusion would be an efficient guide to treatment, and lowering the critical value in order to capture more of those at risk must inevitably select for unnecessary treatment more of those who are not. This is a general problem of any screening technique. There is an additional problem in that a proportion of women are probably immunized by transfusions occurring substantially before delivery, and for them a single Kleihauer test after delivery may not be an appropriate basis of selection.

But in addition to this, the Kleihauer test itself has a built-in limit to its resolution, which depends upon the high sampling variance of the small number of fetal cells upon which it relies. Samples with 5 or more fetal cells will quite frequently represent greater dilutions than 5 cells represent on average, and dilutions which on average give less than 5 cells will, in a substantial proportion of samples, show more than this number. The exact calculation of false positive and false negative rates at any particular level is formally not possible, because it requires prior knowledge of the primary distribution of dilutions in the population, but if the distribution reported by Woodrow et al. (1965) were taken to represent the population from which it was drawn, about $27 \%$ of positives would be false positives and $15 \%$ of critical transfusions would have been missed. These errors would be greater still if the critical level were lowered, and are of course additional to those arising from heterogeneity of responses to transfusions of differing amounts, and those arising from the timing of the transfusion in relation to the test.

Therefore the efficiency of selective treatment may not improve very much beyond that which it has already achieved, and it seems unlikely that more than $50 \%$ of sensitizing pregnancies can be selected by the Kleihauer test, within the confines of a minority of less than $30 \%$ of all susceptible women.

At the present time the problem of treating so high a proportion is aggravated through shortage of material for treatment and of resources for testing, and it has been suggested that initial applications should be limited to first pregnancies, or perhaps to first and second pregnancies.

A series of examinations was carried out, using the model, of the relation between incidence and the application of preventive techniques of varying efficiency at the first pregnancy only. The results are represented in the Fig. in curve B. Once more there is a remarkable linearity of response, and inspection shows that a $30 \%$ efficiency here, reducing the risk from 0.081 to 0.056 , would reduce the incidence from 6.5 per 1000 to about 5.5 per 1000 , and an eventual efficiency of $50 \%$ reduction at the first pregnancy would reduce the incidence to about 4.9 per 1000 .

The model also permits the calculation of yield for effort applied at different birth ranks. In 1960 there were 375 first births per 1000 total births and approximately $8 \%$ of them, that is about 30 , were Rh-positive ABO homospecific infants in Rh-negative mothers. Further developments in selection technique might isolate half of the sensitizing pregnancies among a $20 \%$ minority, when 30 tests and 6 treatments per 1000 total births would reduce the incidence by 1.6 per 1000 . This amounts to 19 tests and 4 treatments per case saved. But at second pregnancies by similar methods each case saved would require 31 women to be screened, and at third pregnancies, over 35. Screening of first and second pregnancies would require 25 tests and perhaps 5 treated for every case saved, and would result in a reduction of total incidence from $6 \cdot 5$ to $4 \cdot 1$.

Knox (1968) has produced evidence showing that it is possible on clinical grounds alone to isolate a group consisting of about $7 \%$ of all pregnancies, this group having a $30 \%$ risk of sensitization and containing about $25 \%$ of all those women in which sensitization occurred. With suitable technical developments there may be scope here for reducing the frequency of significant fetal/ maternal transfusion by $25 \%$, and a test run on the model showed that this would reduce the incidence from $6 \cdot 5$ to $4 \cdot 9$, a result identical with the above application of a developed Kleihauer selection and subsequent treatment, at the first birth rank only.

A combined scheme might give better results. Its eventual capacity might be of the order of $50 \%$ reduction of risk at the first rank and $25 \%$ reduction at subsequent ranks. This might be regarded as optimistic, at least for the immediate short term, but when tested on the model reduced the incidence only to $4 \cdot 1$ per 1000 total births. 


\section{Discussion}

The use of computer models to study the plausibility of premises and the effects of changing them, and to examine the consequences of policy decisions, is now an established technique. It is particularly valuable when the consequences are so complex a function of the premises that a simple mathematical relationship cannot be stated. In general there are two kinds of models, stochastic and deterministic. Stochastic models depend upon probabilistic processes and usually use pseudo random-number generators. They are particularly valuable for studying low intensity events and require to be run many times in order to develop a picture of what is likely to happen. The present model is of the deterministic type and always gives the same results from the same premises.

A complex model with many variables, even a deterministic one, offers such great scope for variation that the same results may be attainable from many different starting points. Consequently, a degree of certainty concerning some of the premises is particularly valuable if combinations of others are to be explored with any confidence. This is why special attention was given to the credentials of the various postulates which were used in constructing the model. The essential facts for the construction of a model of haemolytic disease are a knowledge of the timing and sequencing of the main determinants, an indication of their magnitude, and a reasonable certainty that none has been omitted.

Any policy decision invokes the concept of a model. When the policy decision concerns a population, then it must be a population model whether it be intuitive and implicit, or explicit and exactly stated as in the present case. For the proper evaluation of any large scale therapeutic or prophylactic application, it is necessary not only to know the numbers of people who ought to have been treated and were treated, but also those who ought to have been and were not, and those who ought not to have been and were. Evaluations are required for the consequences of action in each of these three classes and sometimes also among the fourth class, the true negatives (see McKeown (1968) for a more extended discussion).

In the present case, application of the model to the estimated incidence of the disease finds the proportion who ought to be treated, that is $8 \%$ of ABO homospecific $R_{1} r$ pregnancies. The screening procedure itself defines the sum of the true and false positives, and at this point it is possible to enter all the marginal totals of a $2 \times 2$ table setting the proportion selected for treatment against the proportion who ought to be treated. The full picture is constructed by measuring in addition the proportion of pregnancies that were both selected for treatment and in need of treatment. It is found as the product of the proportion isolated by the test, multiplied by the proportion of those isolated who in fact would suffer if not treated (e.g. as in the control groups of the trials mentioned earlier).

The results of application of results such as this, derived from available evidence, forces a pessimistic conclusion. The actual and potential discriminatory efficiency of the Kleihauer test makes it highly unlikely that the disease can be controlled effectively by the treatment of a minority of pregnant women selected in this way.

This is not to say that a worth-while diminution of incidence cannot be obtained. It can. But a really dramatic reduction of the incidence of HDN will only be obtained if very much more accurate discrimination can be achieved, or if the treatment is applied to a majority of women, proably on a non-discriminative basis (except perhaps for dose). This raises new problems of resources and the supply of material which have not yet been faced, together with questions concerning the effectiveness of treatment in an environment for which the trials reported to date do not supply adequate answers.

\section{Summary}

Evidence concerning the location, sequence, timing, and magnitude of the main determinants of haemolytic disease of the newborn is reviewed, and its sufficiency for constructing a model is established.

A working computer model is described, the consequences of which depend upon an input of the population birth rank distribution, the $\mathrm{ABO}$ and rhesus gene frequencies, and risk weightings for $R_{2} r$ and $A B O$ heterospecific pregnancies, and for different birth ranks. The model establishes a relation between the basic risk of sensitization and the incidence of disease in the population, and can operate in either direction.

The model is validated through comparisons between observed and expected birth rank distributions of affected and first affected infants, and confirms previous demonstrations that the risk of sensitization in susceptibles does not vary substantially with birth rank.

Varied inputs to the model are used to indicate the variations of incidence which would result from changing birth rank distributions in the population, from changes in sensitization risk at all birth ranks, and from changes in sensitization risk at selected 
birth ranks, in particular those that might result from the application of prophylactic procedures.

Rhesus iso-immunization may be prevented by treating selected women with anti-D antibodies, but methods of selection are inefficient. Testing on the model showed that the combination of selection and treatment methods presently available was unlikely to control the disease.

\section{REFERENCES}

Clarke, C. A. (1968). Prophylaxis of rhesus Iso-immunisation. Brit. med. Bull., 24, 3.

- et al. (1966). Prevention of rh-haemolytic disease: results of the clinical trial. A combined study from centres in England and Baltimore. Brit. med.f., 2, 907.

Finn, R., Clarke, C. A., Donohoe, W. T. A., McConnell, R. B., Sheppard, P. M., Lehane, D., and Kulke, W. (1961). Experimental studies on the prevention of Rh-haemolytic disease. Brit. med. F., 1, 1486.

Knox, E. G. (1968). Obstetric determinants of rhesus sensitization. $1,433$.
- Murray, S., and Walker, W. (1960). Location of the adventitious factors in haemolytic disease of the newborn. Brit. $\mathcal{F}$. prev. soc. Med., 14, 77.

- immunisation. f. Obstet. Gynaec. Brit. Cwlth, 68, 11.

, and Walker, W. (1957). Nature of the determinants of rhesus iso-immunization. Brit. f. prev. soc. Med., 11, 126.

Levine, P. (1943). Serological factors as possible causes in spontaneous abortions. $\mathcal{f}$. Hered., 34, 71 .

McKeown, T. (1968). In Screening for Medical Care. Published for Nuffield Provincial Hospitals Trust, by Oxford University Press, London. In preparation.

Murray, S., Knox, E. G., and Walker, W. (1965). Haemolytic disease and the rhesus genotypes. Vox Sang. (Basel), 10, 257.

Nevanlinna, H. R., and Vainio, T. (1956). Influence of motherchild ABO incompatibility on rh immunisation. ibid., 1, 26.

Pilkington, R., Knox, E. G., Russell, J. K., and Walker, W. (1966). Foetal-maternal transfusion and rhesus sensitization. $\mathcal{F}$. Obstet. Gynaec. Brit. Cwlth, 73, 909.

Walker, W., Murray, S., and Russell, J. K. (1957). Induction of labour to prevent recurrent stillbirth due to haemolytic disease. Lancet, $1,348$.

Woodrow, J. C., Clarke, C. A., Donohoe, W. T. A., Finn, R McConnell, R. B., Sheppard, P. M., Lehane, D., Russell, S. H., Kulke, W., and Durkin, C. M. (1965). Prevention of rhhaemolytic disease; a third report. Brit. med. F., 1, 279. 